\title{
Computing the Vulnerable Phase in a 2D Discrete Model of the Hodgkin-Huxley Neuron
}

\author{
Dragos Calitoiu, B. John Oommen and Doron Nussbaum \\ Carleton University \\ Canada
}

\section{Introduction}

In several neurological diseases, like essential tremor, the functions of the brain are severely impaired by synchronized processes, in which the neurons fire in a synchronized periodical manner at a frequency closely related to that of the tremor. Stimulation techniques have been developed to desynchronize these neuronal populations. One such technique is the electrical Deep Brain Stimulation (DBS) (Luders, 2004), (Mayberg, 2005) which is performed by administering a permanent high frequency periodic pulse train to the brain by means of so-called depth electrodes. The DBS method was developed empirically, and its mechanism has not yet been understood.

Another stimulation technique is the perturbation with brief stimuli. Clinical results for this technique (some of them are briefly presented in this chapter) prove that a carefully chosen brief pulse applied at a specific time, denoted by the term "vulnerable phase", can annihilate the firing behaviour in the neuron. It is believed that by determining the vulnerable phase of a neuron, the result can be generalized to a population of neurons.

In this context, the first neural model analytically investigated in great detail was the Hodgkin-Huxley $(\mathrm{HH})$ neuron, which exhibits stable periodic solutions for a certain range of constantly applied depolarizing currents.

To study the latter from a variety of perspectives, we shall first present, in Section 1.1, the dynamics of the HH neuron. Then, in Section 1.2, we informally describe its annihilation and stability properties and compare its characteristics with the properties of some of its close "relatives". Finally, in Section 1.3, we shall describe the HH model from the context of the works of Winfree and Guckenheimer.

\subsection{Dynamics of the $\mathrm{HH}$ neuron}

We present now a few considerations about the dynamical properties of the $\mathrm{HH}$ neuron. This neural model can be in one of two states: a resting state and a state that fires in response to certain forms of stimulation. Usually, the neuron is considered to be in a stable mode when it is in a resting state. However, this statement is not universal because there are two stable states associated with this neuron, namely a fixed point and the limit cycle, both of which are stable. One problem to be considered here is the switching of the neuron from one stable mode to the other, which is a phenomenon that can occur without modifying the number and the stability of the equilibria. 
Put in a nutshell, we would like to determine if the HH neuron in 2D is controllable (i.e., if it can be driven from a quiescent state to a spiking state and vice versa). However, it turns out that the general system is unsolvable, the latter being a consequence of three well-known fundamental results, namely Hilbert 16-th Problem, the Poincare-Bendixon Theorem and the Hopf Bifurcation Theorem. These three results are cited to prove that an analytic analysis to obtain the exact representation of the separatrix is not feasible. Having achieved this, we proceed to tackle the problem of concern from a topological perspective, and show that the control is achievable by exciting the system with an appropriate pulse. Not only have we proved the existence of this pulse, but also described its characteristics (amplitude, duration etc.).

From a classical system theory point of view, the stable point of a nonlinear dynamical system may disappear or may lose its stability if a control parameter is changed, depending on the type of bifurcation displayed by the system. In our research, the $\mathrm{HH}$ neuron is considered to be a dynamical nonlinear system whose stable states are not to be radically changed with regard to its stability. We investigate the case when both stable states, namely the fixed point and the limit cycle, co-exist and remain stable. In addition to the fixed points and limit cycles, a 2D system can also possess homoclinic ${ }^{1}$ points, which, in turn, imply the existence of a hyperbolic invariant set on which the $2 \mathrm{D}$ system is chaotic.

In this particular situation, the system is bi-stable, without homoclinic points, and, with a carefully chosen synaptic input, it is possible to switch the behaviour from being resting to one which demonstrates spiking, or from being spiking to a resting (spike annihilation) mode. The goal of this research is to describe the properties of the stimulus that can achieve this switching.

This above stimulus, chosen to be a brief pulse of current, is not a control parameter. Its behaviour affects neither the existence of the fixed points or limit cycles, nor their stability. The control parameter is the strength of the constantly applied current and, during our investigation, it is set to be constant. We argue that injecting a constant current into the axon is not equivalent to injecting a brief pulse of current. In the former, the system can go through a bifurcation of the stable state by changing the existence of the stable states or by affecting their stability. In the latter, however, the system can jump to an alternate location in the state space, which is achieved by the system resetting the initial condition. The neuron is driven to a state of "shock", and consequently, the membrane potential instantly switches

\footnotetext{
1 It can be advantageous to clarify the concepts of points that are homoclinic and heteroclinic. We do this by invoking the following definitions essentially from (Devaney , 2003). Let $p$ be a repelling fixed point, with $f^{\prime}(p)>1$, namely $|f(x)-p|>|x-p|$. We define a local unstable set at $p$, denoted as $W_{l o c}^{u}(p)$, to be the maximal open interval in the neighbourhood of $p$. A point $q$ is said to be homoclinic to $p$ if $q$ $\in W_{l o c}^{u}(p)$ and if there exists $n>0$ such that $f^{n}(q)=p$ (where $f^{n}(x)$ is defined as $f\left(f^{n-1}(q)\right)$ ). The point $q$ is heteroclinic if $q \in W_{l o c}^{u}(p)$ and if there exists $n>0$ such that $f^{n}(q)$ lies on a different periodic orbit. If $p$ has a homoclinic point $q, p$ it is also so-called "snap-back repellor". Since $q$, by definition, lies in the local unstable set in the neighbourhood of $p$, it is possible to define a sequence of pre-images of $q$, each of which lies closer to $p$ in the local unstable set. Thus, the homoclinic point, $q$, together with its backward orbit defined by the pre-images and its forward orbit, is called a homoclinic orbit. This orbit has the property that it tends to the fixed point, $p$, when a "backward iteration" is invoked, and it lands on the same fixed point if a "forward iteration" is invoked.
} 
to a new value. The fixed point, corresponding to the resting state, co-exists with the limit cycle, which corresponds to the spiking state, and the system continues to be bistable. This leads us to the goals of this research: (i) to prove analytically the existence of such stimuli, and (ii) to describe the characteristics of these brief depolarizing shock-stimuli that, when inserted at the appropriate time, can switch the neuron from the spiking to the resting state.

\subsection{The HH neuron: the annihilation perspective}

The annihilation of the firing activity was predicted theoretically by Teorell (Teorell, 1971) for a two-variable model of a sensory pacemaker. He showed that the annihilation of the firing activity can be achieved by using a small brief test pulse injected into the refractory period, just prior to the system attaining to its firing level. Later, the annihilation of the spike train, by using a carefully chosen stimulus, was predicted by Rinzel ${ }^{2}$ (Rinzel, 1980) and also independently by Best (Best, 1979). Rinzel calculated periodic solutions to the spaceclamped $\mathrm{HH}$ equations when a depolarizing current was constantly applied. The computational analysis of Best stated that one could "shock" the HH neuron out of the repetitive mode by using a properly timed instantaneous current pulse. In addition, Guttman, Lewis, and Rinzel (Guttman, 1980) experimentally confirmed that repetitive firing in a space-clamped squid axon, merely stimulated by a suprathreshold step of current, can be annihilated by a brief depolarizing or hyperpolarizing pulse of the proper magnitude, applied at the proper phase. After the resting potential of the axon (whose central compartment was bathed in low $\mathrm{Ca}$ artificial seawater) had reached a steady state, the threshold for repetitive firing was established by a manually triggered stimulation with a step of current, $30 \mathrm{~ms}$ in duration, to avoid overstimulation of the axon. Thereafter, a slightly suprathreshold current step of approximately $30 \mathrm{~ms}$ duration, was used as a bias in order to initiate the repetitive firing. Upon being excited by this bias current, various magnitudes of brief $0.15 \mathrm{~ms}$ perturbations were added at various phases in the period of the response, to investigate the control of repetitive firing. In response to such perturbations, membrane potentials and ionic currents showed damped oscillations that converged towards a steady state. For the non-annihilating perturbations, the repetitive firing of the system resumed with an unaltered frequency, but with a modified phase.

Closely related to the Rinzel model for the HH neuron, is the model due to FitzHughNagumo. Theoretical considerations relevant to the latter have also been derived by Baer and Erneux (Baer, 1986), who studied the phenomena of singular Hopf bifurcations from a basic state to that involving relaxation oscillations. For the model of the FitzHugh-Nagumo neuron, they analyzed the switching from a stable steady state to a stable periodic solution (spike generation) and the reverse situation (spike annihilation). They succeeded in formally explaining both these phenomena.

\subsection{The HH neuron: the Winfree/Guckenheimer perspective}

The annihilation problem that we have solved for a 2-dimension $\mathrm{HH}$ neuron can be viewed from an entirely different perspective. This point of view involves the control of the

\footnotetext{
2 Although the Rinzel model that we have used is a few years old, we do not believe that it is outdated. As far as we know, the Rinzel model is probably the best reported 2-D model for the HH structure. Furthermore, is also well known that increasing the accuracy of the coefficients does not modify the fundamental dynamics of the neuron.
} 
isochrones of a general dynamical system, and in particular, of networks involving neurons akin to the $\mathrm{HH}$ neuron. Historically, the origin of this perspective can be traced to "traditional biology", where Winfree, in his pioneering papers (Winfree, 1974) and (Winfree, 1977) anticipated the existence of a perturbing stimulus that could affect the dynamics of the system. This hypothesis actually resulted from his initial research on fibrillation, which involves the uncontrolled fluttering of the heart, possibly leading to sudden cardiac death. Later, Winfree applied topological concepts to investigate the effects of involving disturbing stimuli that could change the human biological clock expressed, for example, by alternating sleep-wake cycles at almost-regular intervals. He predicted that in order to generate an arrhythmic pattern, a stimulus should be applied at a specific point in the sleep-wake cycle. Winfree further suggested mathematical models for describing this family of behaviours related to biological clocks, and though these models were very pertinent, they also provided a fertile ground for further research because they raised unforeseen topological questions, that were related to phase resetting.

Winfree's research phenomena were subsequently investigated by Guckenheimer (Guckenheimer, 1975), who, on the other hand, described analytically, using the foundational theory of ordinary differential equations, many of the open problems proposed by the former. In particular, he concentrated on the existence and the properties of the above mentioned "isochrones". However, while Winfree's interest was related to biological clocks, Guckenheimer's intention was to establish a methodology for analyzing the stability of the limit cycle, which is a component of the dynamics of biological clocks. From this perspective, and based on the so-called assumption of nondegeneracy, Guckenheimer determined the condition for which two points could be isochrones. He concluded that the existence of isochrones is determined by the flow near the limit cycle, and more specifically, formulated the theorems that involve the intersections of the isochrones of a limit cycle and the neighborhood of its frontiers.

The followings are the three topics proposed by Winfree, and which Guckenheimer proved analytically in (Guckenheimer, 1975): 1. The properties of the isochrone lines: Guckenheimer showed that these are related to a stable manifold in a dynamical system, this being a special case of the Invariant Manifold Theorem. 2. The topology of a stable manifold of a stable limit cycle: Guckenheimer showed that this determines the dimension of its frontier. 3 . The properties of points in the neighbourhood of the frontier that intersects the isochrones.

The last problem involves three distinct directions. The first direction introduced the concept of open-dense sets of vector fields. The second investigation included the concept of generic $^{3}$ subsets. The third theorem used the previous results and proved the existence of dense open subsets of vector fields with the property that every neighbourhood of every point in the frontier meets each isochrone of the limit cycles. Guckenheimer also tested his results experimentally. He stated that the results displayed one of the following two phenomena: (i) The destruction of the oscillation entirely, or (ii) The fact that points arbitrarily close to each another lay on isochrones of every point of a limit cycle. In summary, Guckenheimer's work was conducted so as to analytically characterize the second scenario.

To present our work in this perspective, we, first of all, mention that in our research, we analytically investigate the first scenario. Also, we can formally describe the relation between our work and the Winfree-Guckenheimer research, as follows:

${ }^{3}$ A subset of a topological space is generic if it is a countable intersection of open-dense sets. 
Similarity: Both of approaches investigate the stability of a dynamical system, with the goal of controlling it in the neighbourhood of a limit cycle. The control is achievable by exciting the system with an appropriate pulse, which is invoked when the system is in the neighborhood of the limit cycle. Finally, both Guckenheimer and we demonstrate that the characteristics of the limit cycle determine the effect of the excitation.

Difference: Although the similarities between the works exist, it is prudent for us to highlight the dissimilarities. Our first intention is to prove the existence of the stimulus that is able to entirely destroy the oscillation -- which is an issue that Guckenheimer has not analyzed. To achieve this, we have used the bi-stability property of the $\mathrm{HH}$ neuron, with the goal of annihilating the oscillation, and of forcing the system to move through the stable fixed point. Consequently, we have also investigated analytically the first scenario unearthed by the simulations that Guckenheimer reported. From an analytical point of view, Guckenheimer's work investigated the conditions that maintain the limit cycle to be unaffected by the stimuli. His work is related only to the neighbourhood of the stable limit cycle without investigating a model which contains both a stable limit cycle, a fixed point and a region separating them which includes a separatrix - an unstable limit cycle. Thus, Guckenheimer has not investigated the effect of adding a stimulus with a goal of forcing the system through separatrix so as to reach the fixed point.

In contrast to the previous pieces of work cited above, which validated experimentally or anticipated theoretically that annihilation is possible, we achieve the following:

1. We formally prove that the problem of spike annihilation has a well defined solution.

2. We formally derive the characteristics of the proposed solution.

3. We demonstrate experimentally the validity of the solution (i.e., by numerical simulations).

All of the results are novel, and we thus believe that our analysis of the $\mathrm{HH}$ neuron has practical implications in clinical applications ${ }^{4}$, especially in the case of the desynchronization of neuronal populations.

\subsection{Format of the chapter}

Section 1 presents an overview of the clinical research related to the problem of spike annihilation in $\mathrm{HH}$ neurons. Section 2 contains the dynamical formulation of the problem, namely the bistable neuron, the equations of the system, and its stable and unstable limit cycles. Section 3 investigates the problem of annihilation and presents a formal proof of the existence of the stimulus, and the suggested numerical approach for computing the bifurcation point. Section 4 describes the experiments conducted for determining the properties of the annihilation stimulus, and Section 5 concludes the chapter.

\footnotetext{
${ }^{4}$ A few investigations which are applicable to optimizing the characteristics of the stimuli used to annihilate real NNs have been reported. Two renowned investigators, in this field are Dr. Osorio from University of Kansas - Kansas Medical Center, and Dr. McIntyre from Carleton University, in Ottawa, Canada. The former has been praised for his work in the project titled "Safety, tolerability and efficacy of high-frequency periodic thalamic stimulation in inoperable mesial temporal epilepsy" (Osorio et al., 2005), and the latter is well known for his work in low frequency brain stimulations against kindled seizures (Carrington et al., 2007) and (McIntyre et al., 2005). Unfortunately, their more recent results are not published yet.
} 


\section{The bistabile HH neuron}

In this section we investigate the stability-related characteristics of the $\mathrm{HH}$ neuron. In the previous section, we stated that the $\mathrm{HH}$ neuron can be perceived as a dynamical nonlinear system with two stable equilibria. This is formalized below.

Consider a two-dimensional dynamical system:

$$
\begin{aligned}
& \frac{d V}{d t}=P(V, R) \\
& \frac{d R}{d t}=Q(V, R)
\end{aligned}
$$

where $P(V, R)$ and $Q(V, R)$ are polynomials of real variables $V$ and $R$, and where the corresponding coefficients are real. The fundamental problem associated with the qualitative theory of such systems seems to be the second part of Hilbert's Sixteenth Problem (Gray, 2000), stated as follows:

Specify the configuration and the maximum number of limit cycles that a planar polynomial differential system can have as a function of its degree.

This problem remains unsolved.

It should be mentioned that there are many methods which yield specific results related to the study of limit cycles. However, the above general problem has not been solved, ${ }^{5}$ even for the quadratic systems. Rather, we intend to explore, numerically, the less general system defined by Equations (3) and (4) proposed by Wilson (Wilson, 1999), which, indeed, approximate the Hodgkin-Huxley neuron:

$$
\begin{gathered}
\frac{d V}{d t}=\frac{1}{\tau}\left[-\left(\mathrm{a}_{1}+\mathrm{b}_{1} \mathrm{~V}+\mathrm{c}_{1} \mathrm{~V}^{2}\right)\left(\mathrm{V}-\mathrm{d}_{1}\right)-\mathrm{e}_{1} \mathrm{R}\left(\mathrm{V}+\mathrm{f}_{1}\right)+\mathrm{B}+\sigma\right] \\
\frac{d R}{d t}=\frac{1}{\tau_{R}}\left(-\mathrm{R}+\mathrm{a}_{2} \mathrm{~V}+\mathrm{b}_{2}\right)
\end{gathered}
$$

where $a_{1}, a_{2}, b_{1}, b_{2}, c_{1}, d_{1}, e_{1}, f_{1}, \tau$ and $\tau_{R}$ are constants ${ }^{6}, B$ is the background activity ${ }^{7}$, and $\sigma$ is an excitation stimulus. Apart from deriving certain specific analytic results, we propose to discover, numerically, the number and the positions of the limit cycles.

By introducing Hilbert's Sixteenth Problem as a motivation for the solutions of the system, we argue that the numerical approach to yield the number and the relative positions of the

\footnotetext{
${ }^{5}$ Solutions for specific cases of classes of planar differential equations, such as the Lienard equations, systems having homogeneous components of different degree, homogeneous systems perturbed by a constant system, etc. have been reported. Even in these cases, the solutions only yield the number of limit cycles, but not their specific forms.

6 In their experiments, Wilson (Wilson,1999) set the constants as: $a_{1}=17.81, b_{1}=47.71, c_{1}=32.63, d_{1}=0.55$, $e_{1}=0.55, f_{1}=0.92, a_{2}=1.35, b_{2}=1.03, \tau=0.8 \mathrm{~ms}$ and $\tau_{R}=1.9 \mathrm{~ms}$. The stimulus $\sigma$ was expressed in $\mu \mathrm{A} / 100$, and $V$ was measured in deci-volts. All these values were assigned to mimic real-life brain phenomena. 7 The background activity generates limit cycles in the system. Without this value, the system will converge through the stable spiral point.
} 
limit cycles of the system, described by Equations (3) and (4), is the only reasonable strategy (instead of an analytical one) to tackle the problem.

It is true that there are some theoretical results (Gray, 2000), which can be postulated as theorems, that can be applied for two-dimensional nonlinear systems. But their contributions are only qualitative without being capable of describing the complete picture of the number and the relative positions of the limit cycles. Thus, in the interest of completeness we mention these formal results that can be used to prove that a system described by Equations (3) and (4) has a limit cycle and a bifurcation point.

In our analytical approach, we propose the following:

1. To identify if in the space of the trajectories of the HH neurons there is only a single area corresponding to the spiking behaviour, and only a single area corresponding to the quiescent behaviour.

2. To identify the curve that separates these two areas - also known as the "separatrix". Observe that the knowledge of the equations of the curve can lead us to determine a stimulus that crosses the boundary, from the spiking state area into the quiescent state area. Since the explicit form of the separatrix is not available (and cannot be determined), we intend to use topological arguments to demonstrate the existence of the excitation sought for.

In this vein, after computing the fixed points and analyzing their stability, we shall further investigate the computation of the limit cycles. The first hurdle encountered is the fact that the stable limit cycle that corresponds to the spiking behaviour has a set of equations that cannot be determined analytically. In addition, the curve that separates the two areas is itself a limit cycle, albeit an unstable one, that also can not be computed analytically. Thus, as mentioned earlier, we have opted to prove the existence of the curve that separates the two areas by using only topological arguments. Having achieved this, we shall proceed to solve the original problem, i.e., to prove the existence of the stimulus by using only qualitative aspects of the system. Thus, we shall answer the following: (i) When do the limit cycles occur? and (ii) When is a limit cycle stable or unstable?

To aid us in this endeavour, we shall use the results of the following theorems, first explained informally, and then more formally.

1. The Poincare-Bendixon Theorem. This theorem states that if a system has a long-term trajectory in a two dimensional state space limited to some finite-size region, called its invariant set ${ }^{8}$, the system has a fixed point or a limit cycle. This theorem works only in two dimensions because only in a two-dimensional domain a closed curve separates the space into a region "inside" the curve and a region "outside". Thus, a trajectory starting inside a limit cycle can never get out of it, and a trajectory starting outside can never enter into it.

2. The Hopf Bifurcation Theorem. This theorem describes the birth and the death of a limit cycle. We resort to this result because our task is to prove the existence of an unstable limit cycle (i.e., the separatrix) between the basin of attraction of the attracting fixed point and the basin of attraction of the attracting stable limit cycle. Fortunately, this separatrix, which can only be proven to exist using the Hopf Bifurcation Theorem, is the curve that separates the area that corresponds to the spiking behaviour and the second area that corresponds to the quiescent behaviour.

${ }^{8}$ Any trajectory starting from a point in this region will stay there for all time. 
The reader will observe that as a consequence of these theorems, we can conclude that it is not possible to find the analytical representation of the separatrix, although we can prove its existence.

\subsection{Related Theoretical Foundation}

The first useful Theorem, due to Poincare and Bendixon (Hilborn, 2000), defines the conditions for the existence of a limit cycle.

\section{The Poincare-Bendixon Theorem}

1. Consider a system whose long-term motion of a state point in a two-dimensional state space is limited to some finite-size region;

2. Suppose that this region, say $R$, is such that any trajectory starting within $R$ stays within $R$ for all time ${ }^{9}$.

3. Consider a particular trajectory starting in $R$. There are only two possibilities for that trajectory:

a. The trajectory approaches a fixed point of the system as $t \rightarrow \infty$.

b. The trajectory approaches a limit cycle as $t \rightarrow \infty$.

The Hopf Bifurcation Theorem and a supporting result (referred to as Theorem 0) (Devaney, 2003) presented below, define the conditions for the existence of a stable or unstable limit cycle. The following theorems ${ }^{10}$ are essentially taken from (Devaney, 2003).

Theorem 0 Consider the family of maps $F_{\mu}(z)=\mu z+O(2)$ where $\mu$ is not a $k^{\text {th }}$ root of unity for $k=1, \ldots, 5$. Then there is a neighborhood $U$ of 0 and a diffeomorphism $L$ on $U$ such that the map $L^{-1}{ }^{\circ} F_{\mu}{ }^{\circ}$ $L$ assumes the form $z_{1}=\mu z+\beta(\mu) z^{2} z^{\prime}+O(5)$.

Hopf Bifurcation Theorem Suppose $F_{\lambda}$ is a family of maps depending on a parameter $\lambda$ and satisfying:

i. $\quad F_{\lambda}(0)=0$ for all $\lambda$.

ii. $D F_{\lambda}(0)$ has complex conjugate eigenvalues $\left\{\mu(\lambda), \mu^{\prime}(\lambda)\right\}$ with $|\mu(0)|=1$ and $\mu(0) \neq k^{\text {th }}$ root of unity for $k=1, \ldots, 5$.

iii. $\frac{d}{d \lambda}|\mu(\lambda)|>0$ when $\lambda=0$.

iv. In the normal form given by Theorem 0 , the term $\beta(\mu(0))<0$.

Then there is an $\varepsilon>0$ and a closed curve $\zeta_{\lambda}$ in the form $r=r_{\lambda}(\theta)$, defined for $0<\lambda<\varepsilon$ and invariant under $F_{\lambda}$. Moreover, $\zeta_{\lambda}$ is attracting in a neighborhood of 0 and $\zeta_{\lambda} \rightarrow 0$ as $\lambda \rightarrow 0$.

It is necessary to mention the following two relevant remarks, taken from (Devaney, 2003):

1. The assumption that $\frac{d}{d \lambda}|\mu(\lambda)|>0$ when $\lambda=0$ means that the eigenvalues cross from the inside of the unit circle to the outside as $\lambda$ increases.

2. If we reverse the inequalities (ii), (iii) and (iv) above, the Hopf Bifurcation Theorem still remains valid. However, after the bifurcation, the invariant circle is repelling while the origin is attracting.

The Hopf Bifurcation Theorem indicates that in the parameter space there is a limit cycle. It does not tell us whether this is an unstable limit cycle or an asymptotically stable limit cycle. However, the theorem specifies where in the parameter space we can search to locate a limit cycle behaviour. Thus, although we are not able to provide the equation that describes the limit cycle, we can qualitatively describe it.

\footnotetext{
${ }^{9} \mathrm{R}$ is called an "invariant set" for the system.

10 The notation $O(k)$ means terms of degree $k$ or more.
} 
To render our theoretical consideration meaningful, in the following, we shall derive:

1. The fixed points of the $\mathrm{HH}$ neuron by solving the system of equations described by the isoclines,

2. The Jacobian corresponding to the system described by Equations (3) and (4), at the fixed points,

3. The eigenvalues of the Jacobian, by solving the characteristic equation associated with the Jacobian, and

4. The requirements on the eigenvalues as specified by the Hopf Bifurcation Theorem for identifying the limit cycle.

\subsection{Computing the fixed points}

Consider a system described by Equations (3) and (4). We compute the fixed points by solving the system of equations described by their isoclines. This is formalized in the following Lemma.

Lemma 1 The fixed points of the HH neuron can be obtained by solving a cubic polynomial equation:

$$
\mathrm{x}_{3} \mathrm{~V}^{3}+\mathrm{x}_{2} \mathrm{~V}^{2}+\mathrm{x}_{1} \mathrm{~V}+\mathrm{x}_{0}=0 \text {, }
$$

where: $x_{3}=-c_{1}, x_{2}=-\left(b_{1}+a_{2} e_{1}-c_{1} d_{1}\right), x_{1}=-\left(a_{1}-b_{1} d_{1}+a_{2} e_{1} f_{1}+b_{2} e_{1}\right), x_{0}=a_{1} d_{1}-b_{2} e_{1} f_{1}+B$.

Proof From Equations (3) and (4), we see that the system has two isoclines, specified by the contours: $\frac{d V}{d t}=0$ and $\frac{d R}{d t}=0$, which can be written as:

$$
\begin{gathered}
\frac{1}{\tau}\left[-\left(\mathrm{a}_{1}+\mathrm{b}_{1} \mathrm{~V}+\mathrm{c}_{1} \mathrm{~V}^{2}\right)\left(\mathrm{V}-\mathrm{d}_{1}\right)-\mathrm{e}_{1} \mathrm{R}\left(\mathrm{V}+\mathrm{f}_{1}\right)+\mathrm{B}\right]=0, \\
\frac{1}{\tau_{R}}\left(-\mathrm{R}+\mathrm{a}_{2} \mathrm{~V}+\mathrm{b}_{2}\right)=0 .
\end{gathered}
$$

The background activity $B$ is the control parameter $\beta$ specified in the Hopf Bifurcation Theorem.

The fixed points can be computed as solutions of Equations (6) and (7). By substituting $R$ from Equation (7) as $R=a_{2} V+b_{2}$, and utilizing this value in Equation (6), we obtain the equation:

$$
\mathrm{x}_{3} \mathrm{~V}^{3}+\mathrm{x}_{2} \mathrm{~V}^{2}+\mathrm{x}_{1} \mathrm{~V}+\mathrm{x}_{0}=0,
$$

where the coefficients $x_{3}, x_{2}, x_{1}$ and $x_{0}$ are as defined in the Lemma statement. Hence the Lemma.

Remarks:

1. The roots for the variable $V$ in Equation (5) can be computed for specific values of $B$, the background stimulus, which is constantly applied to obtain a bistable neuron.

2. Using the settings of Rinzel and Wilson (Wilson, 1999), assigned to mimic real-life brain phenomena, Equations (6) and (7) become: 


$$
\frac{1}{\tau}\left[-\left(17.81+47.71 \mathrm{~V}+32.63 \mathrm{~V}^{2}\right)(\mathrm{V}-0.55)-26 \mathrm{R}(\mathrm{V}+0.92)+\mathrm{B}\right]=0
$$

and

$$
\frac{1}{\tau_{R}}(-\mathrm{R}+1.35 \mathrm{~V}+1.03)=0
$$

The fixed points can thus be computed as solutions of Equations (9) and (10), leading to the resulting cubic polynomial equation:

$$
-32.6304 \mathrm{~V}^{3}-64.8632 \mathrm{~V}^{2}-50.6416 \mathrm{~V}+\mathrm{B}-14.8424=0
$$

The roots of the Equation (11) are computed for specific values of $B$, and tabulated in Table 1.

\begin{tabular}{|l|l|l|l|}
\hline B & Root1 & Root2 & Root3 \\
\hline 0 & -0.6979 & $-0.6449+0.4856 \mathrm{i}$ & $-0.6449-0.4856 \mathrm{i}$ \\
\hline 0.025 & -0.6947 & $-0.6465+0.4854 \mathrm{i}$ & $-0.6465-0.4854 \mathrm{i}$ \\
\hline 0.05 & -0.6915 & $-0.6482+0.4852 \mathrm{i}$ & $-0.6482-0.4852 \mathrm{i}$ \\
\hline 0.06 & -0.6902 & $-0.6488+0.4852 \mathrm{i}$ & $-0.6488-0.4852 \mathrm{i}$ \\
\hline 0.065 & -0.6896 & $-0.6491+0.4852 \mathrm{i}$ & $-0.6491-0.4852 \mathrm{i}$ \\
\hline 0.07 & -0.6889 & $-0.6494+0.4851 \mathrm{i}$ & $-0.6494-0.4851 \mathrm{i}$ \\
\hline 0.075 & -0.6883 & $-0.6498+0.4851 \mathrm{i}$ & $-0.6498-0.4851 \mathrm{i}$ \\
\hline 0.08 & -0.6876 & $-0.6501+0.4851 \mathrm{i}$ & $-0.6501-0.4851 \mathrm{i}$ \\
\hline 0.085 & -0.6870 & $-0.6504+0.4851 \mathrm{i}$ & $-0.6504-0.4851 \mathrm{i}$ \\
\hline 0.1 & -0.6850 & $-0.6514+0.4850 \mathrm{i}$ & $-0.6514-0.4850 \mathrm{i}$ \\
\hline 0.125 & -0.6818 & $-0.6530+0.4849 \mathrm{i}$ & $-0.6530-0.4849 \mathrm{i}$ \\
\hline 0.15 & -0.6785 & $-0.6546+0.4848 \mathrm{i}$ & $-0.6546-0.4848 \mathrm{i}$ \\
\hline 0.2 & -0.6720 & $-0.6579+0.4847 \mathrm{i}$ & $-0.6579-0.4847 \mathrm{i}$ \\
\hline 0.25 & -0.6655 & $-0.6612+0.4846 \mathrm{i}$ & $-0.6612-0.4846 \mathrm{i}$ \\
\hline
\end{tabular}

Table 1 . The roots of the value $V$ variable for the fixed points equation of the HH neuron as a function of $B$, the background stimulus. The parameters of the neuron are as advocated in (Wilson, 1999)

3. To consider the real-life settings, we have also computed the corresponding value of $R$ for all the real values of the roots, $V$, namely for Root 1 from Table 2. From this Table, we can deduce the range of values for $R$ that is useful in simulating brain-like phenomena. These values will be used later in this paper. 


\begin{tabular}{|l|l|l|}
\hline $\mathrm{B}$ & Root1 $(\mathrm{V})$ & $\mathrm{R}=\mathrm{R}(\mathrm{V})$ \\
\hline 0 & -0.6979 & 0.0878 \\
\hline 0.025 & -0.6947 & 0.0922 \\
\hline 0.05 & -0.6915 & 0.0965 \\
\hline 0.06 & -0.6902 & 0.0982 \\
\hline 0.065 & -0.6896 & 0.0958 \\
\hline 0.07 & -0.6889 & 0.1000 \\
\hline 0.075 & -0.6883 & 0.1008 \\
\hline 0.08 & -0.6876 & 0.1017 \\
\hline 0.085 & -0.6870 & 0.1025 \\
\hline 0.1 & -0.6850 & 0.1052 \\
\hline 0.125 & -0.6818 & 0.1096 \\
\hline 0.15 & -0.6785 & 0.1046 \\
\hline 0.2 & -0.6720 & 0.1140 \\
\hline 0.25 & -0.6655 & 0.1228 \\
\hline
\end{tabular}

Table 2. The values of $R$ obtained for a real root of the fixed points as computed for a particular value of $B$. The parameters of the neuron are as advocated in (Wilson, 1999)

\subsection{Computing the Jacobian}

We now consider a Jacobian-based analysis of the $\mathrm{HH}$ neuron, formalized in the following Lemma.

Lemma 2 The Jacobian matrix of the system representing the HH neuron is given by:

$$
\mathrm{J}(\mathrm{V}, \mathrm{R})=\left(\begin{array}{cc}
\mathrm{y}_{12} \mathrm{~V}^{2}+\mathrm{y}_{11} \mathrm{~V}+\mathrm{y}_{10} & y_{21} V+y_{20} \\
y_{30} & y_{40}
\end{array}\right)
$$

where $y_{12}=-\frac{1}{\tau} 3 c_{1}, y_{11}=-\frac{1}{\tau}\left(2 b_{1}+2 c_{1} d_{1}+a_{2} e_{1}\right), y_{10}=-\frac{1}{\tau}\left(a_{1}+b_{1} d_{1}+e_{1} b_{2}\right), y_{21}=-\frac{1}{\tau} e_{1}, y_{20}=-\frac{1}{\tau} f_{1}, y_{30}=-$ $\frac{1}{\tau_{R}} a_{2,}$ and $y_{40}=-\frac{1}{\tau_{R}}$

Proof We know from the theory of dynamical systems that the Jacobian matrix of the system is :

$J(V, R)=\left(\begin{array}{cc}\frac{\partial V(V, R)}{\partial V} & \frac{\partial V(V, R)}{\partial R} \\ \frac{\partial R(V, R)}{\partial V} & \frac{\partial R(V, R)}{\partial R}\end{array}\right)$. Evaluating each of these components yields: 


$$
\begin{gathered}
\frac{\partial V(V, R)}{\partial V}=\frac{\partial\left[\frac{1}{\tau}\left[-\left(a_{1}+b_{1} V+c_{1} V^{2}\right)\left(V-d_{1}\right)-e_{1} R\left(V+f_{1}\right)+B\right]\right]}{\partial V}= \\
=\frac{1}{\tau}\left[-3 \mathrm{c}_{1} \mathrm{~V}^{2}-\left(2 \mathrm{~b}_{1}+2 \mathrm{c}_{1} \mathrm{~d}_{1}\right) \mathrm{V}-\left(\mathrm{a}_{1}+\mathrm{b}_{1} \mathrm{~d}_{1}\right)-\mathrm{e}_{1} \mathrm{R}\right] \\
\frac{\partial V(V, R)}{\partial R}=\frac{\partial\left[\frac{1}{\tau}\left[-\left(a_{1}+b_{1} V+c_{1} V^{2}\right)\left(V-d_{1}\right)-e_{1} R\left(V+f_{1}\right)+B\right]\right]}{\partial R}=-\frac{1}{\tau} e_{1}\left(V+f_{1}\right), \\
\frac{\partial R(V, R)}{\partial V}=\frac{\partial\left[\frac{1}{\tau_{R}}\left(-R+a_{2} V+b_{2}\right)\right]}{\partial V}=\frac{1}{\tau_{R}} a_{2} \\
\frac{\partial R(V, R)}{\partial R}=\frac{\partial\left[\frac{1}{\tau_{R}}\left(-R+a_{2} V+b_{2}\right)\right]}{\partial R}=-\frac{1}{\tau_{R}} .
\end{gathered}
$$

However, Equation (7) can be used to eliminate $R$ from the partial derivatives. By achieving this, and omitting the laborious algebraic steps, the result follows.

Remarks:

1. Observe that the Jacobian $J$ is not dependent on $B$. However, it is clear that $J$ can be evaluated at each fixed point, which, in turn, is dependent on $B$.

2. Using the same settings of Rinzel and Wilson (Wilson, 1999), the Jacobian matrix of the "real-life" HH neural system becomes:

$$
\begin{aligned}
J(V, R)= & \left(\begin{array}{ll}
\frac{\partial V(V, R)}{\partial V} & \frac{\partial V(V, R)}{\partial R} \\
\frac{\partial R(V, R)}{\partial V} & \frac{\partial R(V, R)}{\partial R}
\end{array}\right), \frac{\partial V(V, R)}{\partial V}=-122.36 V^{2}-74.40 V+10.55-32.5 R ; \\
& \frac{\partial V(V, R)}{\partial R}=-32.5 V-29.9 ; \frac{\partial R(V, R)}{\partial V}=0.71053 ; \frac{\partial R(V, R)}{\partial R}=-0.52632 .
\end{aligned}
$$

As mentioned in the proof of the Lemma, Equation (10) can be used to eliminate $R$ from the partial derivatives and thus, the Jacobian becomes:

$$
J(V)=\left(\begin{array}{cc}
-122.36 V^{2}-118.28 V-22.937 & -32.5 V-29.9 \\
0.71053 & -0.52632
\end{array}\right)
$$




\subsection{Finding the bifurcation point}

We shall now consider the problem of finding the neuron's bifurcation point by using the dynamical matrix of the system. This value of the bifurcation point is used to "set" the neuron so as to render it to be bi-stable.

Theorem 1 A HH neuron obeying the Equations (3) and (4) has a bifurcation point if and only if a root of the equation

$$
\frac{1}{\tau}\left[-3 c_{1} V^{2}-\left(2 b_{1}+2 c_{1} d_{1}\right) V-\left(a_{1}+b_{1} d_{1}\right)-e_{1} R\right]-\frac{1}{\tau_{R}}=0 \text { satisfies the inequality } V>-f_{1}-\frac{1}{e_{1}} \frac{1}{\tau_{R}} .
$$

Proof It is well known that for the bifurcation point, the roots of the characteristic equation, computed from the Jacobian, are purely imaginary. It is also well known that a quadratic equation $\mathrm{x}^{2}-S \mathrm{x}+P=0$ has imaginary roots if:

Condition 1: $S=0$,

Condition 2: $P>0$,

where $S$ and $P$ are the sum and the product of the roots, respectively.

Consider the Jacobian of the $\mathrm{HH}$ neuron as given by Lemma 2. Applying Condition 1 to this Jacobian generates the equation:

$$
\frac{1}{\tau}\left[-3 c_{1} V^{2}-\left(2 b_{1}+2 c_{1} d_{1}\right) V-\left(a_{1}+b_{1} d_{1}\right)-e_{1} R\right]-\frac{1}{\tau_{R}}=0 .
$$

This equation has two roots, say $V_{1}$ and $V_{2}$. The problem now is one of verifying whether $V_{1}$ and $V_{2}$ satisfy Condition 2. This in turn implies that for $V_{1}$ and $V_{2}$ :

$$
\frac{1}{\tau_{R}} \frac{1}{\tau}\left[-3 c_{1} V^{2}-\left(2 b_{1}+2 c_{1} d_{1}\right) V-\left(a_{1}+b_{1} d_{1}\right)-e_{1} R\right]+\frac{1}{\tau_{R}} \frac{1}{\tau} e_{1}\left(V+f_{1}\right)>0 .
$$

We can rewrite this inequality using the observation that $V_{1}$ and $V_{2}$ are solutions to the equation corresponding to Condition 1, namely: $\frac{1}{\tau}\left[-3 c_{1} V^{2}-\left(2 b_{1}+2 c_{1} d_{1}\right) V-\left(a_{1}+b_{1} d_{1}\right)-e_{1} R\right]=\frac{1}{\tau_{R}}$.

Using this relation, Condition 2 becomes: $\frac{1}{\tau}+\frac{1}{\tau_{R}} \frac{1}{\tau} \mathrm{e}_{1}\left(\mathrm{~V}+\mathrm{f}_{1}\right)>0$.

We know that $\tau$ and $\tau_{R}$ are time constants, being positive. We make a convention that $e_{1}$ is also a positive constant. With these considerations, Condition 2 can be rewritten in a new form as: $V>-f_{1^{-}} \frac{1}{e_{1}} \frac{\tau}{\tau_{R}}$. The theorem follows since whenever these constraints are satisfied, we obtain purely imaginary roots.

Remarks:

1. As before, using the same settings of Rinzel and Wilson (Wilson, 1999), Condition 1 applied to the Jacobian generates the equation $-122.36 \mathrm{~V}^{2}-118.28 \mathrm{~V}-22.937-0.52632=0$, whose roots are -0.6879 and -0.2788 . It is easy to verify whether either of these roots satisfy the constraint specified by Theorem 1 . Observe that the first root, $V=-0.6879$, 
satisfies the Condition 2 that is equivalent to $V>-0.9361$, implying that the $\mathrm{HH}$ neuron has a bifurcation point.

2. From Equation (11), we can compute the value of $B$ that corresponds to the root $V=0.6879$. This value ${ }^{11}$, of $B=0.0777$, generates a bifurcation in the system.

3. The second root, -0.2788 , does not have any biological significance, being distant from the resting potential of the neuron.

4. The values of the roots (and the corresponding stability consequences) are tabulated in Table 3 as a function of $B$. Examining this table, we can conclude (using the notation of the Hopf Bifurcation Theorem) that $\alpha=0.0777$. Thus, if $B<0.0777$ (namely, $\beta<\alpha$ ) the system has an stable spiral point. If $B>0.0777$, the stable spiral point becomes unstable and the system has a stable limit cycle. The value $B=0.0777$ is a subcritical or hard Hopf bifurcation point. The system has an unstable limit cycle for $B<0.0777$, and this is a point that is not observable in the real world due to its instability. It is only possible to detect the consequences of its presence.

\begin{tabular}{|l|l|l|l|l|}
\hline $\mathrm{B}$ & $\mathrm{V}_{\text {equilib }}$ & $\lambda_{1}$ & $\lambda_{2}$ & \\
\hline 0 & -0.6979 & $-0.2565+2.2485 \mathrm{i}$ & $-0.2565-2.2485 \mathrm{i}$ & $\mathrm{S}$ \\
\hline 0.025 & -0.6947 & $-0.1731+2.2534 \mathrm{i}$ & $-0.1731-2.2534 \mathrm{i}$ & $\mathrm{S}$ \\
\hline 0.05 & -0.6915 & $-0.0909+2.2554 \mathrm{i}$ & $-0.0909-2.2554 \mathrm{i}$ & $\mathrm{S}$ \\
\hline 0.06 & -0.6902 & $-0.0579+2.2555 \mathrm{i}$ & $-0.0579-2.2555 \mathrm{i}$ & $\mathrm{S}$ \\
\hline 0.065 & -0.6896 & $-0.0909+2.2554 \mathrm{i}$ & $-0.0909-2.2554 \mathrm{i}$ & $\mathrm{S}$ \\
\hline 0.07 & -0.6889 & $-0.0909+2.2554 \mathrm{i}$ & $-0.0909-2.2554 \mathrm{i}$ & $\mathrm{S}$ \\
\hline 0.075 & -0.6883 & $-0.0100+2.2548 \mathrm{i}$ & $-0.0100-2.2548 \mathrm{i}$ & $\mathrm{S}$ \\
\hline 0.08 & -0.6876 & $+0.0075+2.2543 \mathrm{i}$ & $+0.0075-2.2543 \mathrm{i}$ & $\mathrm{U}$ \\
\hline 0.085 & -0.6870 & $+0.0225+2.2537 \mathrm{i}$ & $+0.0225-2.2537 \mathrm{i}$ & $\mathrm{U}$ \\
\hline 0.1 & -0.6850 & $+0.0721+2.2514 \mathrm{i}$ & $+0.0721-2.2514 \mathrm{i}$ & $\mathrm{U}$ \\
\hline 0.125 & -0.6818 & $+0.1504+2.2456 \mathrm{i}$ & $+0.1504-2.2456 \mathrm{i}$ & $\mathrm{U}$ \\
\hline 0.15 & -0.6785 & $+0.2299+2.2372 \mathrm{i}$ & $+0.2299-2.2372 \mathrm{i}$ & $\mathrm{U}$ \\
\hline 0.2 & -0.6720 & $+0.3825+2.2138 \mathrm{i}$ & $+0.3825-2.2138 \mathrm{i}$ & $\mathrm{U}$ \\
\hline 0.25 & -0.6655 & $+0.5300+2.1820 \mathrm{i}$ & $+0.5300-2.1820 \mathrm{i}$ & $\mathrm{U}$ \\
\hline
\end{tabular}

Table 3. Eigenvalues of the Jacobian computed from the real root of the fixed point equation obtained with particular values of the background stimulus $B$. Last column describes the stability of the fixed points, namely $S$ (stable) and $U$ (unstable)

\footnotetext{
11 The more exact value is 0.07773267 and it is obtained for $V=-0.687930$ and $R=0.101295$. The Largest Lyapunov exponent for this Hopf bifurcation is 1.000287e-002. For his neural model, Cooley et al. (Cooley et al., 1965) found a value of $0.0765(7.65 \mu \mathrm{A})$ for the value of $B$. By increasing the stimulus further, he obtained finite trains of shortening duration, and finally, at higher intensities, claimed to obtain the annihilation.
} 


\subsection{The Stable and Unstable Limit Cycles}

If we consider $B$ to be a control parameter, we can analytically compute the fixed point, which, for certain values of $\sigma$, leads to a spiral stable point and, for other values of $\sigma$, leads to an unstable spiral point. The behaviour around a specific value, namely the change of the stability of the fixed point, induces the concept of a subcritical (hard) Hopf bifurcation.

Let us focus on the issue of the limit cycles themselves. By plotting the evolutions of the numerical solutions of the system (Equations (3) and (4)), we discover that for the settings of Rinzel and Wilson (Wilson, 1999), there is a stable limit cycle to the right of the bifurcation point. To identify a hypothetical unstable limit cycle, we can modify the system's equations to make time run "backwards". The modification, which consists of rendering the sign of the two constants, $\tau$ and $\tau_{R}$, to be negative, changes the unstable limit cycle to become asymptotically stable. In this way, by using a numerical method, we can identify the position of a second limit cycle, which happens to be unstable. The stable spiral point is surrounded by this unstable limit cycle which, in turn, acts as a separatrix defining a basin of attraction for the stable point.

In Figures 1 and 2 we present the stable and unstable limit cycles, together with the isoclines $\frac{d V}{d t}=0$ and $\frac{d R}{d t}=0$. The trajectory starts at the point indicated by ' 1 ' and follows the arrowed curves. Observe that in the case of Figure 1, the trajectory of the HH neuron follows the stable limit cycle, and in Figure 2, the trajectory follows the unstable limit cycle. Figure 3 depicts the bifurcation diagram. When $B$ is increased from the resting value, the steady state remains asymptotically stable and the spikes are generated only after the bifurcation point is reached by increasing the value of $B$. In other words, the $\mathrm{HH}$ neuron indicates spiking at $B=$ 0.0777 , and the spiking process continues for all values of $B>0.0777$.

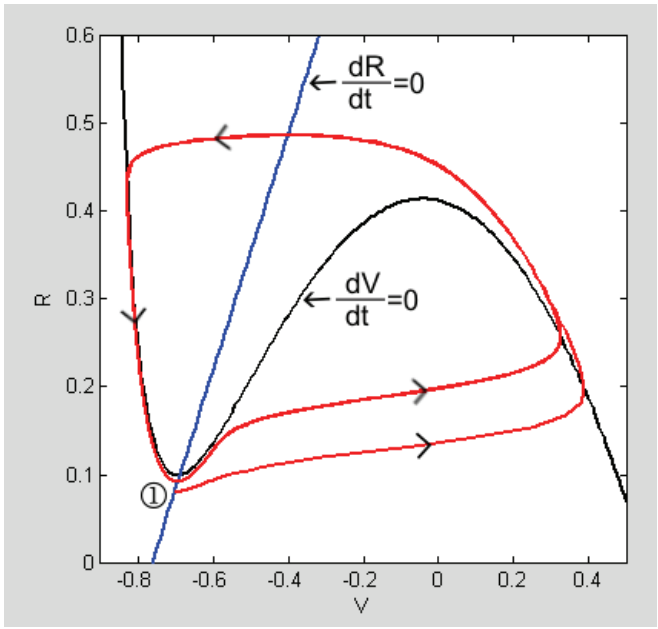

Figure 1. The phase space representing the stable limit cycle and the resulting isoclines $\left(\frac{d V}{d t}=0\right.$ and $\left.\frac{d R}{d t}=0\right)$ obtained by using Rinzel and Wilson settings for the $\mathrm{HH}$ neuron. The starting point, (represented by ' 1 ') is $V_{0}=-0.7$, and $R_{0}=0.08$. In addition, $B=0.08$ 


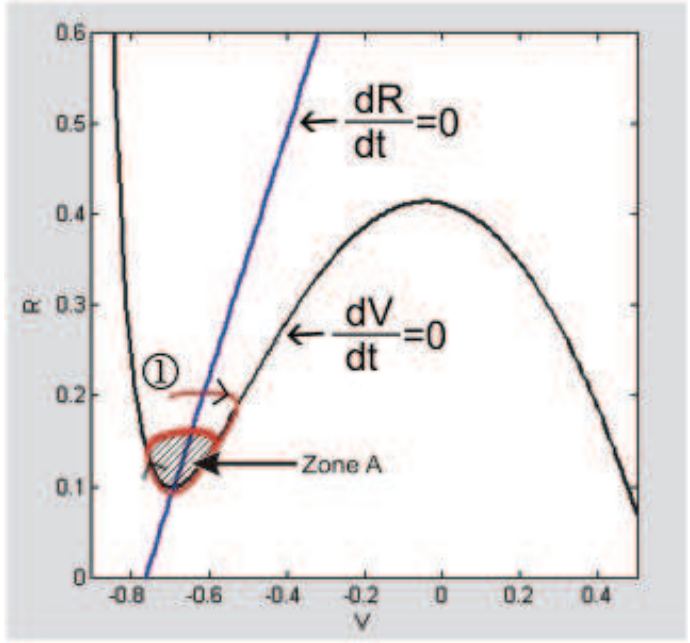

Figure 2. The phase space representing the unstable limit cycle and the resulting isoclines $\left(\frac{d V}{d t}=0\right.$ and $\left.\frac{d R}{d t}=0\right)$ obtained by using Rinzel and Wilson settings for the $\mathrm{HH}$ neuron. The starting point, (represented by ' 1 ') is $V_{0}=-0.7$, and $R_{0}=0.2$. In addition, $B=0.08$

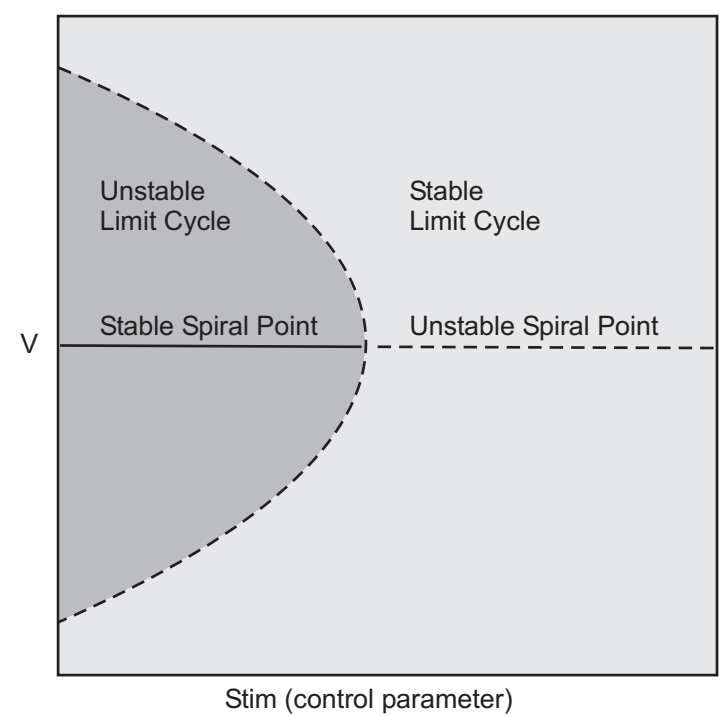

Figure 3. The bifurcation diagram for the system specified in Figures 1 and 2 . The variable $B$ is the control parameter. We consider $B$ as a background stimulus that generates a bi-stable neuron 


\section{The problem of annihilation}

The problem of the annihilation of spikes for the $\mathrm{HH}$ neuron involves moving the state of the system, by using a pulse stimulus, from outside a particular zone (denoted as Zone $\mathrm{A}$ ) to being inside $Z_{\text {Zne }}$, where Zone $e_{\mathrm{A}}$ is a basin of attraction of the stable spiral point which is described by an unstable limit cycle. For example, if the system is characterized by the settings specified by Rinzel and Wilson (Wilson, 1999), Zone $\mathrm{A}_{\mathrm{A}}$ is contained in the region given by $V \in[-0.6,-0.8]$ and $R \in[0.1,0.15]$, as depicted in Figure 2. Figure 4 contains all the steady states of the system, including the stable spiral point, and the stable and unstable limit cycles.

The success of the annihilation process depends on four crucial issues:

1. What should be the initial point $(V, R)$ for the system to exhibit annihilation?

2. When should the pulse stimulus, $\sigma$, be applied to the system to annihilate it?

3. What should the amplitude of the pulse stimulus be for the annihilation to be achieved?

4. What should the duration of the pulse stimulus be for the annihilation to be achieved?

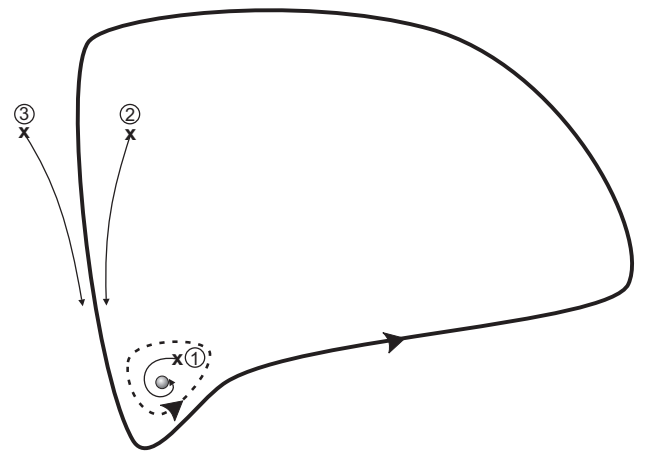

Figure 4. The stable fixed point, the stable limit cycle, and the unstable limit cycle (the separatrix given by the dashed line) are represented together. If the system starts in State 1, it will move towards the stable fixed point. If it starts in State 2 or State 3, it will converge to the stable limit cycle

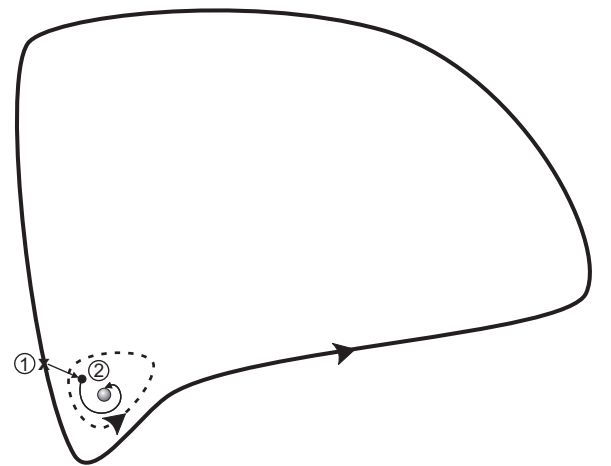

Figure 5. The annihilation process for the system specified in Figures 1 and 2. If the system starts in a carefully chosen configuration at State 1 on the stable limit cycle, the system can be driven to State 2 by applying a carefully chosen stimulus. From this state, it will then go to the stable fixed point 
The solution of the annihilation problem consists of determining a stimulus which adequately responds to all the above questions.

We now formally prove that the problem of spike annihilation is well-defined, and propose an algorithm for finding a solution to it. In addition, we also study the solution of annihilating the spikes by using multi-stimuli. Finally, we investigate the inverse problem, namely that of spike generation (see Figure 6).

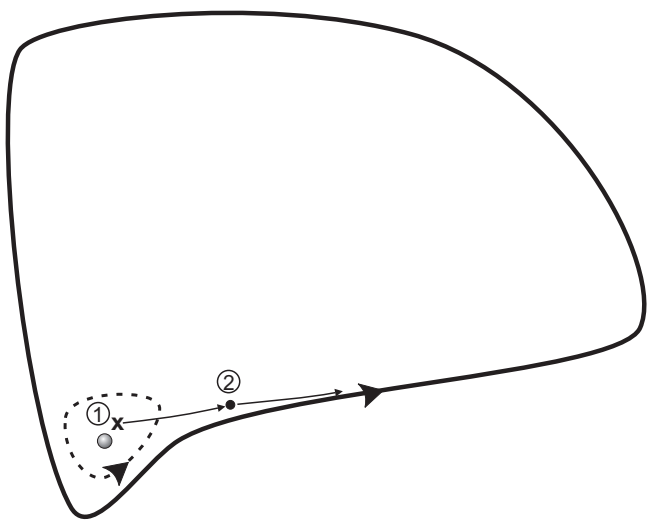

Figure 6. The spike generation process for the system specified in Figures 1 and 2 . If the system starts in a stable fixed point or at State 1, in the close neighborhood of the stable fixed point, the system can be driven to State 2, by applying a specific stimulus, and, from this state, it will go further toward the stable fixed point

The two problems are clarified in Figures 5 and 6 . In Figure 5 we present the annihilation process. If the system starts in a carefully chosen configuration at State 1 on the stable limit cycle, the system can be driven to State 2 by applying a carefully chosen stimulus. From this state, it will then go to the stable fixed point. Similarly, in Figure 6, we depict the spike generation process. If the system starts in a stable fixed point or in State 1, in the close neighborhood of the stable fixed point, the system can be driven to State 2 by applying a specific stimulus, and, from this state, it will go further toward the stable fixed point.

We propose to solve the problem of annihilation from two perspectives:

Problem 1 We plan to analytically demonstrate that the spike annihilation problem has a well-defined solution.

The strategy of solving Problem 1 consists of:

a. Computing the steady states.

b. Analyzing the stability of the steady states.

c. Computing the bifurcation points and the bifurcation diagram.

d. Computing the stable and unstable limit cycles.

e. Analyzing the existence of the stimulus that can annihilate the system.

Problem 2 We intend to numerically compute the characteristics of the stimuli that achieve annihilation, for the settings of Rinzel and Wilson (Wilson, 1999).

The strategy of solving Problem 2 consists of:

a. Proposing an algorithm for computing the moment of insertion, the magnitude, and the duration of the stimulus used to annihilate the system.

b. Analyzing the problem for the case when there are multiple stimuli. 


\subsection{The NN Neuron Annihilation Theorem}

Since we are interested in annihilating the spikes, we shall demonstrate that this can be done by invoking a discretized 12 time model. To achieve this, first of all, we rewrite the dynamical system of equations for a bi-stable model of the $\mathrm{HH}$ neuron in a discrete-time manner as:

$$
\begin{gathered}
V[n+1]=V[n]+\frac{1}{\tau}\left[-\left(a_{1}+b_{1} V[n]+c_{1} V^{2}[n]\right)\left(V[n]-d_{1}\right)-e_{1} R[n]\left(V[n]+f_{1}\right)+B+\sigma\right], \\
R[n+1]=R[n]+\frac{1}{\tau_{R}}\left(-R[n]+a_{2} V[n]+b_{2}\right)
\end{gathered}
$$

The general Theorem of Annihilation is formally written below.

Theorem 2 (HH Neuron Annihilation) Consider a system described by its discretized dynamical equations:

$$
\left(\begin{array}{l}
V[n+1] \\
R[n+1]
\end{array}\right)=\left(\begin{array}{l}
V[n] \\
R[n]
\end{array}\right)+\left(\begin{array}{l}
f_{1}(V[n], R[n]) \\
f_{2}(V[n], R[n])
\end{array}\right)+\underline{S}[n], n=0,1, \ldots
$$

where $f_{1}$ and $f_{2}$ specify the unexcited dynamics, and $\underline{S}[n]$ is the excitation applied to the system. If the system has a stable limit cycle, a stable spiral point and an unstable limit cycle which separates the fixed point and the stable limit cycle, then, there exists an excitation function $\underline{S}[n]$, which equals 0 everywhere except at a specific point $(V[0], R[0])$ on the stable limit cycle, at which point S[0] has the value $[A, 0]^{T}$ for a duration of one iteration, and which when applied to the system, forces it from the stable limit cycle to the stable spiral point.

Proof Consider the system defined by Equation (14), which has the excitation $\underline{S}[n]$. Analyzing the Jacobian of the system, we observe that it has the same form ${ }^{13}$ as the one corresponding to the continuous case. Thus, all the qualitative results obtained in the previous Section are also applicable for the discrete time approach, and thus, the system has a stable fixed point, a stable limit cycle and an unstable limit cycle (also known as a separatrix).

12 A continuous-time approach cannot be invoked to prove this theorem because, by virtue of its
relation to Hilbert's Sixteenth Problem, it is not known how we can compute the explicit solutions for
the system of equations.
${ }^{13}$ The Jacobian of the system is obtained by computing the partial derivative with respect to the state
variables without involving time (continuous or discrete). If the system variable $u$ is expanded
infinitesimally around a quiescent point $u_{0}$ as $u=u_{0}+\Delta u$, the continuous system will lead to
$\frac{d u}{d t}=F(u)$ and the discrete system will lead to $u_{n+1}=F\left(u_{n}\right)$. By dropping the quadratic and higher order terms in $\Delta u$, we can obtain for each of these two systems: $\frac{d \Delta u}{d t}=D F\left(u_{0}\right) \Delta u$ and $\Delta u_{n+1}$ $=D F\left(u_{0}\right) \Delta u_{n}$, respectively. Observe that the Jacobian in both cases has the same form. 


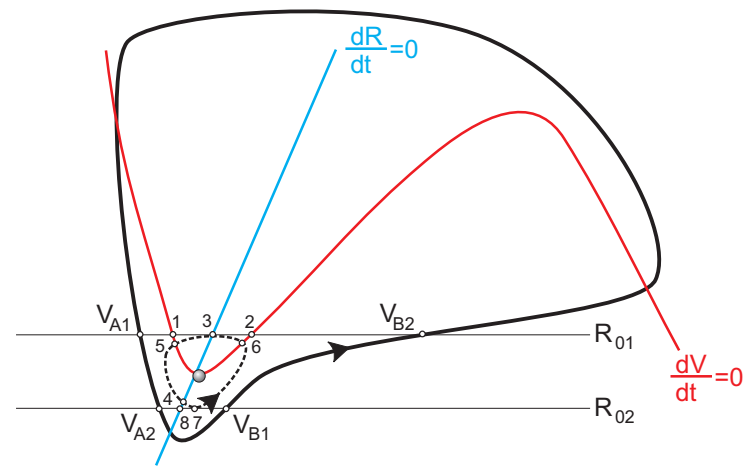

Figure 7. The stable spiral point, the stable and the unstable limit cycle for the bi-stable $\mathrm{HH}$ neuron

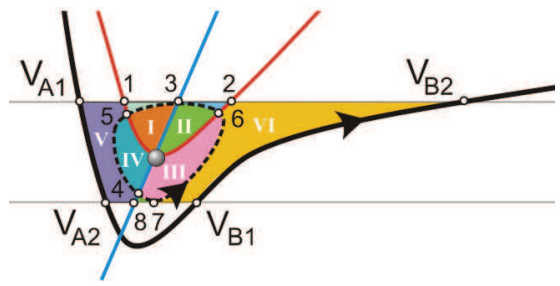

Figure 8. A zoom-in of the Figure (7), namely the phase space of the bi-stable HH neuron. The regions $A_{O u t, 1}$ and $A_{O u t, 2}$ correspond to Area $V$ and Area VI, respectively. The regions $A_{I n, 1}, A_{I n, 2}, A_{I n, 3}$, and $A_{I n, 4}$ correspond to Area I, Area II, Area III, and Area IV, respectively

For the purpose of proof, we define three distinct areas in the state space, as depicted by Figure 8:

1. We denote $A_{\text {In }}$ as the region corresponding to the basin of attraction of the stable fixed point, bordered by the separatrix.

2. We observe two regions outside the separatrix, that can have as their boundaries the tangents in the maximum and minimum ' $R$ ' points on the separatrix, the stable limit cycle and the isoclines. We denote them as:

a) $\mathrm{A}_{\mathrm{Out}, 1}$ : The region where $V[\mathrm{n}+1]>V[\mathrm{n}]$ and $R[\mathrm{n}+1]<R[\mathrm{n}]$, and

b) $\mathrm{A}_{\mathrm{Ou}, 2}$ : The region where $V[\mathrm{n}+1]>V[\mathrm{n}]$ and $R[\mathrm{n}+1]>R[\mathrm{n}]$.

Let us denote the intersection between the tangents in the maximum and minimum ' $R$ ' points on the separatrix, and the stable limit cycle (see Figure 8) as $V_{\mathrm{A} 1}, V_{\mathrm{A} 2}, V_{\mathrm{B} 1}, V_{\mathrm{B} 2}$. The sequence of these points corresponds to the time evolution on the stable limit cycle.

Within the discrete-time model of computation, the problem of annihilation involves proving that there exists a stimulus $A$ which, when applied between $V_{A 1}$ and $V_{A 2}$ or between $V_{B 1}$ and $V_{B 2}$, moves the system into the basin of attraction of the stable fixed point, namely within $A_{I n}$. Observe that if the system is within this region, it is inside the separatrix, and it will thus converge to the fixed point. Indeed, it suffices to show that this input can be applied for a single time unit.

Consider the scenario in which the system is on an initial point $V[0]$ between $V_{A 1}$ and $V_{A 2}$. Since the stable limit cycle and the separatrix are non-intersecting, there exists a positive "distance" $d_{0}$ between $V[0]$ and the separatrix. We intend to determine a value of $A$ that 
moves the system from $(V[0], R[0])$ to an arbitrary point in $A_{I n}$. Clearly, the magnitude $A$ has to satisfy the condition :

$$
(V[1]-V[0])>d_{0}
$$

Computing $V[1]$ as a function of $V[0]$ we have: $V[1]=V[0]+f_{1}(V[0])+A$.

The condition (15) becomes:

$$
\left(V[0]+f_{1}(V[0])+A-V[0]\right)>d_{0} \Rightarrow\left(f_{1}(V[0])+A\right)>d_{0} \Rightarrow A>d_{0}-f_{1}(V[0])
$$

We now invoke the monotonic property of the function $V[n]$, that corresponds to the portion of the state space below the isocline, where $V[n+1]>V[n]$, namely in $A_{I n}$. Here, the term $f_{1}(V[n])=V[n+1]-V[n]$ is positive. We thus see that there exists a value of $A$, satisfying the condition (16), that moves the initial point of the system between $V_{A 1}$ and $V_{A 2}$, to be in $A_{I n}$. We have now to evaluate the sign of the expression $\left[d_{0}-f_{1}(V[0])\right]$. Starting from $(V[0], R[0])$ on the stable limit cycle, with $V[0]$ between $V_{A 1}$ and $V_{A 2}$, we know that, without adding the $A$ stimulus, the next point $(V[1], R[1])$ will also be on the stable limit cycle. The difference between $V[1]$ and $V[0]$ is exactly $f_{1}(V[0])$. In this context, $f_{1}(V[0])$ will satisfy the condition $f_{1}(V[0])<d_{0}$, because there is no intersection between the limit cycle and the unstable limit cycle (described by the separatrix). We have now thus proved that $\left[d_{0}-f_{1}(V[0])\right]>0$. Thus, $A$ is a positive value satisfying $A>d_{0}-f_{1}(V[0])$.

The analogous rationale can be used if the initial point $V[0]$ is between $V_{B 1}$ and $V_{B 2}$. In this case, there exists a distance $d_{1}$ (a positive value) between $V[0]$ and the separatrix. We intend again to find a value of $A$ that moves the system into region $A_{I n}$. The magnitude $A$ has to satisfy the condition:

$$
(V[0]-V[1])>d_{1}
$$

Observe also that this part of the state space, (also below the isocline), corresponds to $V[\mathrm{n}+1]>V[\mathrm{n}]$, and, thus, the term $f_{1}(\mathrm{~V}[\mathrm{n}])=V[\mathrm{n}+1]-V[\mathrm{n}]$ is also positive.

Computing $V[1]$ and $R[1]$ as a function of $V[0]$ and $R[0]$ we have: $V[1]=V[0]+f_{1}(V[0])+A$.

The condition (17) thus becomes:

$$
\left(V[0]-\mathrm{V}[0]-f_{1}(V[0])-A>d_{1} \Rightarrow A<-d_{1}-f_{1}(V[0])\right.
$$

Observe that both $d_{1}$ and $f_{1}(V[0])$ are positive quantities, and thus the term $\left[-\mathrm{d}_{1}-\mathrm{f}_{1}(V[1])\right]$ is a negative value. We have thus proved that there exists a value of $A$ that moves the initial point of the system from being between $V_{B 1}$ and $V_{B 2}$, to be within $A_{I n}$.

Since both these cases are exhaustive, the theorem is proved.

Comments:

1. For each interval $\left[V_{A 1}, V_{A 2}\right]$ or $\left[V_{B 1}, V_{B 2}\right]$ it is possible to choose a value $V[0]$ that corresponds to a particular time instant in the phase space. This time instant can be described as a percentage of the total period of time of the spike. For each chosen $V[0]$, there is a value $\mathrm{d}_{0}$ with a corresponding magnitude $A$ of a unit time stimulus, determined by the conditions (16) or (18).

2. The above proof shows that for any neuron described by Equation (14), there exists an unit time stimulus with the magnitude $A$ satisfying the property that, if it is applied in any place on the limit cycle between $V_{A 1}$ and $V_{A 2}$ or between $V_{B 1}$ and $V_{B 2}$, it will annihilate the spiking behaviour. The problem of annihilation has also a solution for the case when the stimulus is longer then the unit of time. In this case, we need to define in 
the state space four regions inside the separatrix (see Fig. 8), that can be bordered by the isoclines of the system, namely :

a) $A_{I n, 1}$ with the property $V[\mathrm{n}+1]<V[\mathrm{n}]$ and $R[\mathrm{n}+1]<R[\mathrm{n}]$;

b) $A_{[n, 2}$ with the property $V[\mathrm{n}+1]<V[\mathrm{n}]$ and $R[\mathrm{n}+1]>R[\mathrm{n}]$;

c) $A_{I n, 3}$ with the property $V[\mathrm{n}+1]>V[\mathrm{n}]$ and $R[\mathrm{n}+1]>R[\mathrm{n}]$;

d) $A_{I n, 4}$ with the property $V[\mathrm{n}+1]>V[\mathrm{n}]$ and $\mathrm{R}[\mathrm{n}+1]<R[\mathrm{n}]$.

The duration of the stimulus and its magnitude will determine if the system will move from the stable limit cycle, namely from a point in $\left[V_{A 1}, V_{A 2}\right]$ to $A_{I n, 1}$ or to $A_{I n, 4}$, both of them via $A_{\mathrm{Out}, 1}$. The same determination has to be made if the system has to move from a point in $\left[V_{B 1}, V_{B 2}\right]$ to $A_{I n, 2}$ or to $A_{I n, 3}$ via $A_{O u t, 2}$.

\subsection{The numerical approach}

In order to discover the properties of the stimulus which achieves the spikes annihilation, we have also opted to simulate this numerically. To do this, we have to work towards controling the model, namely, to move the system to a bi-stable state, in the neighborhood of the bifurcation point. All these steps will be discussed in the next Section.

\section{Experiments}

In this Section, the analytical results described in Section 3 are experimentally evaluated to verify their validity, and to explore the state space characteristics for each parameter of the annihilation stimulus. If a background stimulus $B$ is applied to create a train of spikes, we demonstrate that it is possible to annihilate the limit cycle with an additional brief stimulus, and to move the system from a stable limit circle to an unstable spiral point.

The solution to this problem has to respond to the following questions:

1. What is the amplitude of the stimulus?

2. What is the suitable phase when the stimulus should be applied?

3. How long should the stimulus be?

4. Is it possible to apply two successive pulses instead of only a single one, in which the phase specification is not so precise? Would this pair of two successive pulses possess the property that they would together be able to annihilate the spikes if the first one, by itself, could not?

In order to analyze the effect of the stimulus, we have to choose initial values for $V$ and $R$. We have studied this for various numerical settings, but present only one scenario here, in the interest of brevity. In Figure 9, we present an example of train spikes that we propose to annihilate with a stimulus. This train of spikes started from $V=-0.7043$ and $R=0$, and was generated with $B=0.08$. In addition, Figure 10 illustrates the corresponding Phase Space of the bi-stable neuron.

In Figure 11, we observe an example of annihilation, where the duration of the train of spikes is $100 \mathrm{~ms}$. In Figure 12, we present the phase space for the bi-stable neuron. Figure 13 is an example of an unsuccessful annihilation observed using a stimulus $\sigma=0.2$, applied at the time instant $3.4 \mathrm{~ms}$ from the beginning of the simulation.

From the bifurcation diagram, we chose the background stimuli $B$ to be between 0.68 and 0.7 . These stimuli generate a spike train. We here chose $V=-0.7043$ and $R=0$ as initial values for the subsequent simulations. 


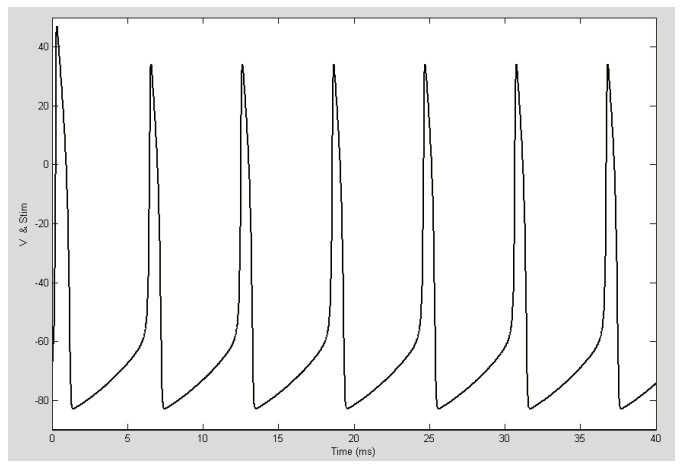

Figure 9. The train of the spikes generated with $B=0.08$

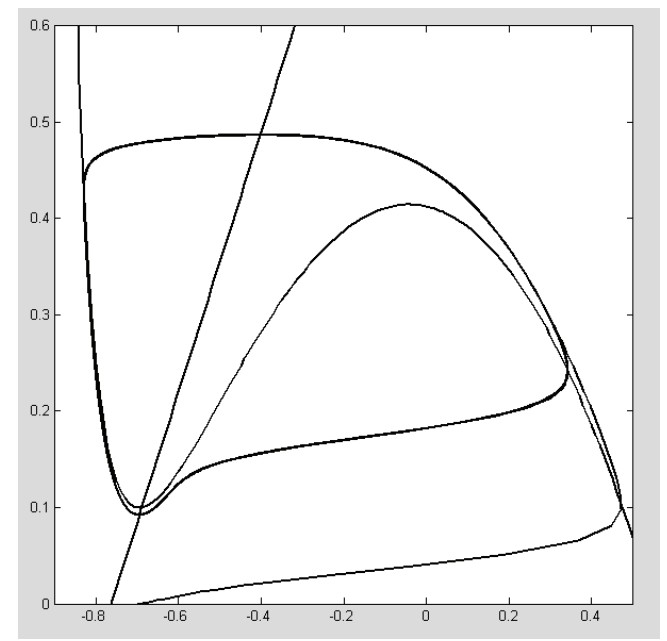

Figure 10. The phase space of the train of the spikes generated with $B=0.08$

For an additional stimulus $\sigma$, namely, a pulse of $0.1 \mathrm{~ms}$ duration ${ }^{14}$, we identified its position of insertion and its amplitude. In Table 4, we present the range of values for $\sigma$ (the minimum and the maximum values) for which we can annihilate the spikes. Each range is computed for different times of insertion of the stimulus (from $3.0 \mathrm{~ms}$ to $4.4 \mathrm{~ms}$ ) and for different values of the quantity $B$. The neuron exhibited spikes only for a range of $B$, which spanned values from 0.68 to $0.70 \mu \mathrm{A} / 100$. The results from Table 4 are depicted in Figure 14 . From this simulation we can conclude that:

1. The neuron spikes only for a specific range of values of $B$;

2. If the neuron generates spikes, these can be annihilated with particular stimuli found in the area plotted in Figure 14.

${ }^{14}$ We will analyze later the effect of the duration of the pulse. 


\begin{tabular}{|l|l|l|l|l|l|l|}
\hline \multirow{2}{*}{$\mathrm{ms}$} & \multicolumn{3}{|l|}{$\mathrm{B}(0.68)$} & \multicolumn{2}{l|}{$\mathrm{B}(0.69)$} & $\mathrm{B}(0.70)$ \\
\cline { 2 - 7 } & $\sigma_{\min }$ & $\sigma_{\max }$ & $\sigma_{\min }$ & $\sigma_{\max }$ & $\sigma_{\min }$ & $\sigma_{\max }$ \\
\hline 3.0 & 0.4 & 1.54 & & & & \\
\hline 3.1 & 0.14 & 1.57 & & & & \\
\hline 3.2 & 0.06 & 1.47 & 0.47 & 1.15 & & \\
\hline 3.3 & 0.28 & 1.34 & 0.19 & 1.23 & 0.50 & 0.97 \\
\hline 3.4 & 0.014 & 1.21 & 0.09 & 1.17 & 0.21 & 1.08 \\
\hline 3.5 & 0.008 & 1.09 & 0.05 & 1.06 & 0.11 & 1.02 \\
\hline 3.6 & 0.005 & 0.97 & 0.03 & 0.95 & 0.062 & 0.93 \\
\hline 3.7 & 0.003 & 0.85 & 0.018 & 0.84 & 0.03 & 0.83 \\
\hline 3.8 & 0.002 & 0.74 & 0.016 & 0.73 & 0.027 & 0.72 \\
\hline 3.9 & 0.002 & 0.63 & 0.01 & 0.63 & 0.02 & 0.62 \\
\hline 4.0 & 0.002 & 0.53 & 0.008 & 0.53 & 0.016 & 0.52 \\
\hline 4.1 & 0.002 & 0.45 & 0.007 & 0.44 & 0.015 & 0.43 \\
\hline 4.2 & 0.002 & 0.35 & 0.007 & 0.34 & 0.015 & 0.33 \\
\hline 4.3 & 0.002 & 0.25 & 0.008 & 0.25 & 0.017 & 0.25 \\
\hline 4.4 & 0.002 & 0.16 & 0.011 & 0.15 & 0.024 & 0.14 \\
\hline
\end{tabular}

Table 4. The amplitude and the moment of insertion of the stimulus $\sigma$ in order to annihilate the spikes

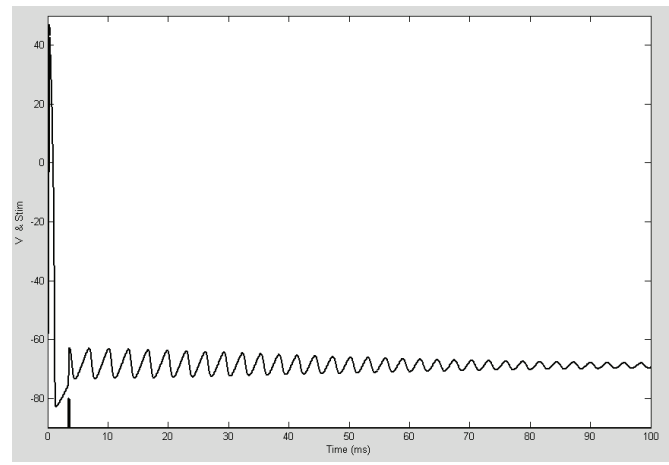

Figure 11. The annihilation of the train of spikes. The presentation is made for $100 \mathrm{~ms}$ 


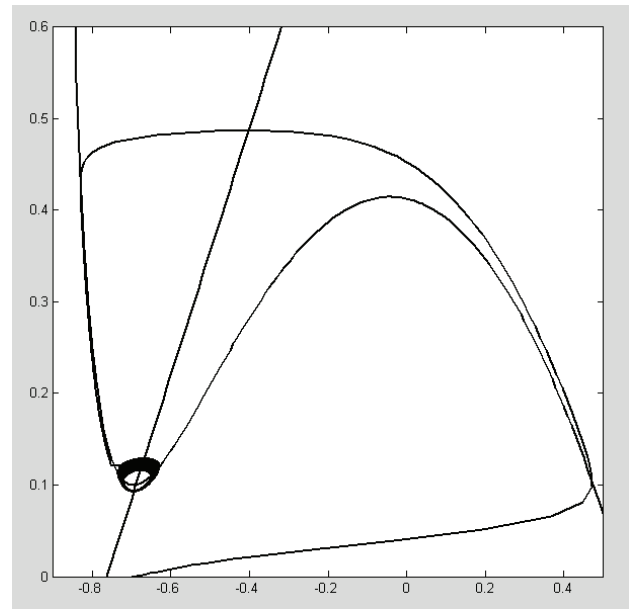

Figure 12. The phase space of a system with the train of spikes annihilated by a stimulus $\sigma$. The presentation is made for $40 \mathrm{~ms}$

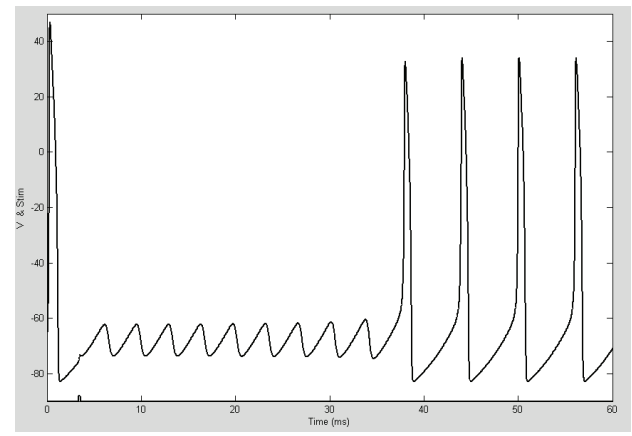

Figure 13. An example of an unsuccessful attempt to annihilate the spikes by using a stimulus $\sigma$ applied at a time instant of $3.4 \mathrm{~ms}$

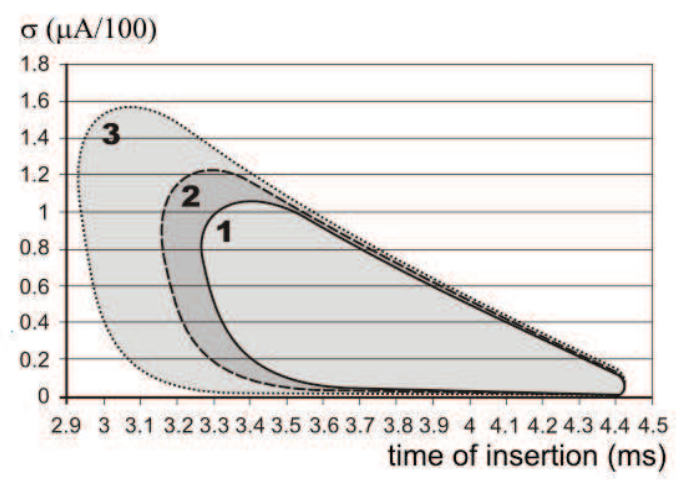

Figure 14. The three areas for the three different values for the background, $B$, namely 0.70 (Area 1), 0.69 (Area 2), and 0.68 (Area 3) 
Consider now the problem of finding the vulnerable phase of the neuron, namely the duration of the period of the signal when the stimulus can be inserted in order to annihilate spikes.

For a value of $\sigma=0.7$, we see from Figure 14 that the length of the vulnerable phase is between $3 \mathrm{~ms}$ to $4.4 \mathrm{~ms}$. Since the period is $6 \mathrm{~ms}$, the neuron has an interval of $23.33 \%$ of its period where one can insert a proper stimulus to achieve this annihilation.

The reader can observe that for the experimental results reported, we conducted experiments with three different background stimuli in order to generate a bi-stable neuron, namely with $B=0.68, B=0.69$, and $B=0.70$. For all these values, we present in Figure 14 three areas, namely those depicted by Area 1, Area 2, and Area 3. Fortunately, there seems to be an inclusion relationship between these three areas, namely Area 1 is included in Area 2 and Area 3. Consider now the scenario when a population of neurons from the brain receives a constant stimulus with the magnitude having a minimum value of 0.68 for an interval of time. If the task is to annihilate the spiking behaviour of this population of neurons, the imprecision of determining the background stimulus will not affect our selection of the annihilation stimulus. Choosing one with a magnitude corresponding to the minimum background is successful because such a stimulus is common for all background stimuli greater than this minimum one. For example, the area corresponding to $B=1$ includes the area corresponding to the minimum $B=0.68$. This observation makes the choice of a successful annihilation stimulus easier and independent from the precision of determining $B$.

\subsection{The duration of the stimulus}

A brief analysis of the duration of the stimulus would be beneficial. Such a study would help the reader to decide on the best stimulus to be used to achieve the annihilation. To do this, we explore numerically the range of the duration for a stimulus with magnitude equal to unity. For example, if the time of insertion is set to be at $3.5 \mathrm{~ms}$, the range of the duration of the stimulus can be between $0.0099 \mathrm{~ms}$ and $0.1095 \mathrm{~ms}$, independent of the value of B whose value lies between 0.68 and 0.7 .

We mention that this numerical determination was made in a scenario with an a priori setting of the amplitude of the stimulus. In the general case, we want to apply a stimulus with the duration $\delta_{1}$, smaller than the period of firing of the $\mathrm{HH}$ neuron, for example $6 \mathrm{~ms}$. One possible approach to determine the magnitude of the stimulus is by using a heuristic search. An algorithm for computing a solution contains, first of all, the determination of the number of iterations corresponding to the duration of the stimulus, namely $\mathrm{k}=\frac{\delta_{1}}{\delta_{2}}$, where $\delta_{0}$ is the numerical time unit, typically chosen to be very small.

Next, we have to determine, by a heuristic search, the magnitude, $A$, of the stimulus, by estimating the pairs $\left(V_{0}, R_{0}\right)$ and $\left(V_{\text {new }}, R_{\text {new }}\right)$. This involves using $\mathrm{k}$ and the rule of computing the new initial point, proposed in Section 3.1, namely:

$$
\begin{gathered}
V_{\text {new }}=V_{0}+f_{1}\left(V_{\mathrm{k}}\right)+\ldots+f_{1}\left(V_{0}\right)+k^{*} A ， \\
R_{\text {new }}=R_{0}+f_{2}\left(R_{\mathrm{k}}\right)+\ldots+f_{2}\left(R_{0}\right) .
\end{gathered}
$$

The reader should observe that we have presented here the difficult scenario of achieving the spike annihilation with a pulse of duration $\mathrm{k}^{*} \delta_{0}$. In a clinical application, the solution to the problem of annihilation can be reduced to the computation of the magnitude of a brief pulse, where it is sufficient to set $\mathrm{k}=1$. 


\subsection{How many stimuli?}

We analyze now the problem of using two successive stimuli to annihilate the spike train. This pair of successive pulses has the property that the first pulse is not able to annihilate the spike train by itself. However, in order to cumulate the effects of the stimuli, we have to apply a second pulse so as to have the distance in time between stimuli less than the period of firing of the $\mathrm{HH}$ neuron. Intuitively, if the distance between the stimuli is more than a period, the neuron does not memorize the effect of the first stimulus, which we can also verify.

To simulate this in a realistic setting, we assume that we don't know exactly the juncture in time where we can insert the single stimulus in order to annihilate the spikes, namely the range $\left[\theta_{1}, \theta_{2}\right]$. Thus, we intend to insert two stimuli, having the same amplitude and a temporary distance between them, $\delta_{2}$.

Consider the general problem of inserting the first stimulus anywhere in the range of $\left[\theta_{1}-\varepsilon, \theta_{1}\right.$ $+\varepsilon$ ]. By proposing $\delta_{2}$, the temporary distance between them, we intend to devise an algorithm for the heuristic search of the magnitude of the stimuli.

We set the initial magnitude to a small value. The proposed algorithm, then, has three phases:

i. The first step consists of the computation of the pair $\left(V^{1}{ }_{\text {new }}, R^{1}{ }_{\text {new }}\right)$, after the insertion of the first stimulus;

ii. The second step consists of the computation of the pair $(V, R)$, knowing the pair $\left(V^{1}\right.$ new, $\left.R 1_{\text {new }}\right)$, and the number of iterations required by dividing $\delta_{2}$ by the integration time unit.

iii. The third step consists of the computation of the $\left(V^{2}{ }_{\text {new }}, R^{2}\right.$ new $)$, after the insertion of the second stimulus.

If, after this computation, the new point, namely $\left(V^{2}{ }_{\text {new }}, R^{2}\right.$ new $)$, is not a point inside the unstable limit cycle, we increase the initial magnitude with a quantity $\Delta A$, and we repeat all the above three steps. Clearly, it is a straightforward "Hypothesize and Test" heuristic search scheme for the amplitude of the stimuli. The problem will lead to (or not lead to) a solution, depending on the values of $\varepsilon$ and $\delta_{2}$.

In this way, a pair of stimuli with a carefully chosen amplitude and a fixed temporal distance between them can annihilate a train of spikes by decreasing the accuracy of the place of insertion. The first stimulus is chosen with a random magnitude and is inserted into the neuron. At his juncture, we will not know if this stimulus is successful or not in annihilating the spikes. By taking into consideration a posteriori its magnitude and its moment of insertion, we want to be able to set the properties of the second stimulus so as to annihilate the neuron, if the first stimulus was not successful. In this way, we can extrapolate the problem of applying, in a range of time $\left[\theta_{1}-\varepsilon_{1}, \theta_{2}+\varepsilon_{2}\right]$, a pair of two stimuli with the same amplitude $\mathrm{A}$, with a duration equal to unity and a temporary distance between them of $\delta_{2}$.This leads us to a scheme for computing the properties of the second stimulus when the first stimulus is given. However, the problem of determining $A, \varepsilon_{1}$ and $\varepsilon_{2}$, having only $\delta_{2}$ is still open.

By simulations, for the setting described in (Wilson, 1999), we showed that, for a Background of 0.7 , the range $\left[\theta_{1}, \theta_{2}\right]$ is $3.3 \mathrm{~ms}-4.4 \mathrm{~ms}$ (see Table 4 ). We have tested the effect of a pair of two successive stimuli, the first being applied too early, at $3.2 \mathrm{~ms}$, and the second one, at 4.2 ms. Both stimuli have the same amplitude, 0.7. From Table 4, we observe that the successful annihilation can be achieved with a stimulus having the amplitude between 0.015 and 0.33 . 
In the scenario with the first stimulus being inserted too early, the second one was successful in annihilating the spikes at an amplitude of 0.7. Thus, the presence of the first stimulus in a zone outside of Area 1 (see Figure 14) has a positive effect, allowing the second stimulus to achieve annihilation, also from a zone outside of Area 1.

In Figure 15, we present an example of a successful annihilation by using two stimuli with amplitude of 0.7 , the first one being applied at $3.2 \mathrm{~ms}$, and the second one at $4.2 \mathrm{~ms}$, where the neuron has a background stimulus, $B$, equal to 0.7 .

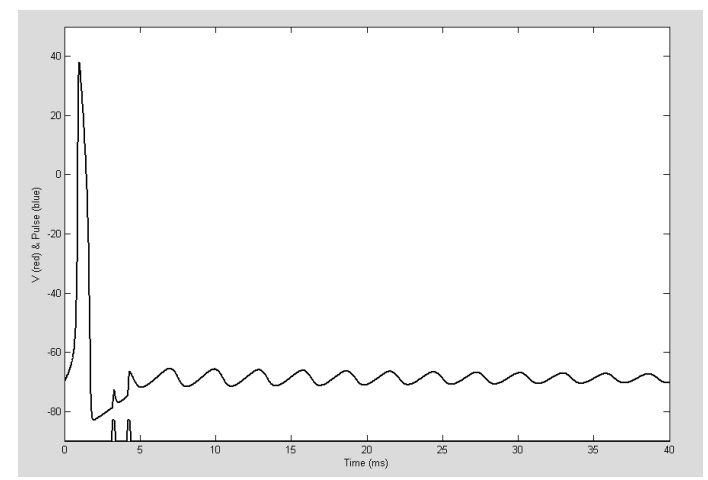

Figure 15. The annihilation using two stimuli with amplitude 0.7 , the first applied at $3.2 \mathrm{~ms}$ and the second applied at $4.2 \mathrm{~ms}$

\subsection{Spike Generation}

We present here, for sake of the completeness of the modeling approach, a particular case involving spike generation. In Section 2, we stated that the $\mathrm{HH}$ neuron has two equilibria, a fixed point and the limit cycle, both of them co-existing and being stable. Thus, the $\mathrm{HH}$ neuron is bi-stable and, with a carefully chosen synaptic input, it is possible to switch its behaviour from a resting state to a spiking (spike generation) state or from a spiking state to a resting state (which is the spike annihilation phenomenon). The spike annihilation problem was solved in Section 3. Here, we study the generation of the spiking behaviour.

If the neuron is in the resting state, namely in the stable fixed point, there are no changes in time. Thus, there is no preference for the moment when one can insert a stimulus in order to move the point $(V, R)$ to be outside of the unstable limit cycle. The stimulus will modify only the $V$ component of the pair $(V, R)$. Observe that in this case there are two limit values for this problem: a positive minimum value that moves the system to the left side of the fixed point and outside of the unstable limit cycle, and a negative maximum value that moves the system to the right side of the fixed point while being outside of the unstable limit cycle.

Again, to demonstrate that this can be achieved, we tested by simulations the scenario when the system is in an fixed point $(\mathrm{V}=0.6889, \mathrm{R}=0.1)$, for $\mathrm{B}=0.7$. In this situation, the system remains in this fixed point forever. If, however, at anytime we excited the system with a single pulse, for example one with the amplitude equal to unity, the system started to oscillate forever. This phenomenon is portrayed in Figure 16. We observe here that, without any background activity, namely with $\mathrm{B}=0.0$, the system cannot oscillate, because it is not bistable. 


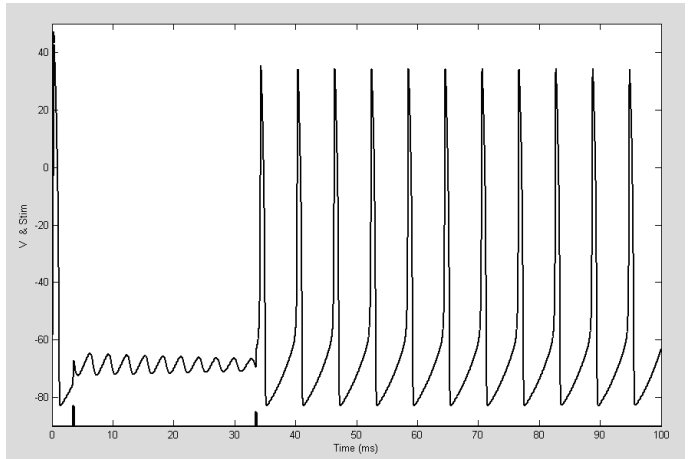

Figure 16. The annihilation and the generation of a new train of spikes. The first stimulus has an amplitude of 0.7 and is applied at $3.5 \mathrm{~ms}$. The second stimulus has an amplitude of 0.5 and is applied at $33.5 \mathrm{~ms}$. The value of $B$ is 0.7

\section{Discussion and Conclusions}

This chapter discussed the $\mathrm{HH}$ neuron and formally derived various properties of its stability. It also described the first (to the best of our knowledge) reported formal proof that the problem of spike annihilation has a well defined solution, and presented an algorithm for computing the properties of the stimulus. We elaborated, in Sections 3 and 4, all the details of this algorithm. We add that the method of perturbation with brief stimuli differs from the classical approach of modifying the control parameter and changing the Jacobian of the system. In our approach, we keep the system bi-stable all the time, and our task is to switch between these two states without modifying their stability.

To conclude, in this chapter we have analytically proved the existence of the brief current pulse that annihilates the spikes of the $\mathrm{HH}$ neuron, when delivered to its repetitively firing state, and have also analyzed the properties of this pulse, namely, the range of time when it can be inserted, its magnitude, and its duration. In addition, we have also studied the solution of annihilating the spikes by using two successive stimuli, where the first one is unable to annihilate the spikes by itself. We have also briefly investigated the inverse problem to annihilation, namely, the spike generation problem, and proposed a straightforward numerical solution.

\section{References}

Baer, S.M. \& Erneux, T. (1986). Singular Hopf bifurcation to relaxation oscillations, SIAM Journal of Applied Mathematics, 46:1986, pp. 721-739, ISSN 0036-1399

Best, E.N. (1979). Null space in the Hodgkin-Huxley equations. Biophysical Journal, 27:1979, pp. 87-104, ISSN 0006-3495

Carrington, C.; Gilby, K.L., \& McIntyre, D.C. (2007). Effect of low frequency stimulation on amygdala kindled afterdischarge thresholds and seizure profile in Fast and Slow kindling rat strains. Epilepsia, 48:2007, pp. 1604-1613 (2007) ISSN 0013-9580

Cooley, J.; Dodge, F., \& Cohen, H. (1965). Digital computer solutions for excitable membrane models. Journal of Cellular and Comparative Physiology, 66:1965, pp. 99108, ISSN 0021-9541 
Devaney, R.L (2003). An Introduction to Chaotic Dynamical Systems (second edition). Westview Press, ISBN-10: 0813340853, Colorado

Gray, J.J. (2000). The Hilbert Challange. Oxford University Press, ISBN-10: 0198506511, USA

Guckenheimer, J. (1975). Isochrons and phaseless sets. Journal of Mathematical Biology, 1:1975, pp. 259-273, ISSN 0303-6812

Guttman, S.L. \& Rinzel, J. (1980). Control of repetitive firing in squid axon membrane as a model for a neuron-oscillator, Journal of Physiology, 305:1980, pp.377-395, ISSN 0022-3751

Hilborn, R.C. (2000). Chaos and nonlinear dynamics (second edition), Oxford University Press, ISBN-10: 0198507232, USA

Luders, H.O. (ed.) (2004). Deep brain stimulation and epilepsy. Martin Dunitz, an imprint of the Taylor and Francis Group, ISBN-10: 1841842591, USA

Mayberg, H.S.; Lozano, A.M., Voon, V., McNeely, H.E., Seminowicz, D., C, Hamani, J. M. \& Schwalb, S.H.K.(2005): Deep brain stimulation for treatment-rezistant depression, Neuron, 45:2005, pp. 651-660 ISSN 0896-6273

McIntyre, D.C.; Carrington, C., \& Gilby, K.L. (2005). The ying-yang of low frequency sinewave stimulation in amygdalia kindled rats, American Epilespy Society Abstr. 2005, ISSN 15357957

Osorio, I., Frei, M.G., Wilkinson, S.B., Sunderam, S., Bhavaraju, N.C., Graves, N., Schaffner, S.F., Peters, T., Johnson, A.M., DiTeresi, C.A., Ingram, J., Nagaraddi, V., Overman, J., Kavalir, M.A., \& Turnbull, M. (2001) Seizure Blockage with Automated ClosedLoop Electrical Stimulation: A Pilot Study. Epilepsia, 42:2001, ISSN 0013-9580

Rinzel, J. (1980). Numerical calculation of stable and unstable periodic solutions to the Hodgkin-Huxley equations, Mathematical Biosciences, 49:1980, pp. 27-59, ISSN 00255564

Teorell, T. (1071). A biophysical analysis of mechano-electrical transduction, in Handbook of Sensory Physiology, vol 1, 1971, pp. 291-339 W.R.Loeswenstein ed, Springer Verlag, ISBN 35400514449, Berlin

Wilson, H. (1999). Spikes decisions and actions: Dynamical foundations of Neuroscience, Oxford University Press, ISBN-13:9780198524304, USA

Winfree, A. (1974). A Patterns of Phase Compromise in Biological Cycles. Journal of Mathematical Biology, 1:1974, pp.73-95, ISSN 0303-6812

Winfree, A. (1977): Phase Control of Neural Pacemakers, Science, 197:1977, pp. 761-763, ISSN 0036-8075 


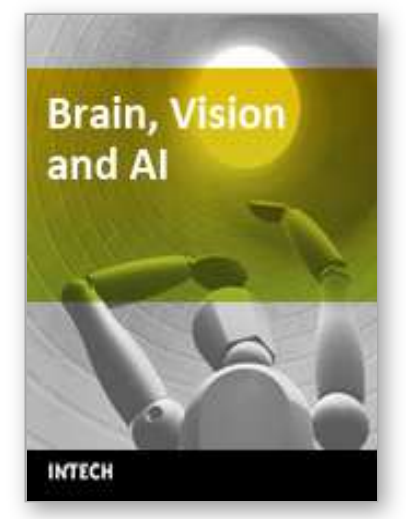

\author{
Brain, Vision and Al \\ Edited by Cesare Rossi
}

ISBN 978-953-7619-04-6

Hard cover, 284 pages

Publisher InTech

Published online 01, August, 2008

Published in print edition August, 2008

The aim of this book is to provide new ideas, original results and practical experiences regarding service robotics. This book provides only a small example of this research activity, but it covers a great deal of what has been done in the field recently. Furthermore, it works as a valuable resource for researchers interested in this field.

\title{
How to reference
}

In order to correctly reference this scholarly work, feel free to copy and paste the following:

Dragos Calitoiu, B. John Oommen and Doron Nussbaum (2008). Computing the Vulnerable Phase in a 2D Discrete Model of the Hodgkin-Huxley Neuron, Brain, Vision and Al, Cesare Rossi (Ed.), ISBN: 978-953-761904-6, InTech, Available from:

http://www.intechopen.com/books/brain_vision_and_ai/computing_the_vulnerable_phase_in_a_2d_discrete_m odel_of_the_hodgkin-huxley_neuron

\section{INTECH}

open science | open minds

\author{
InTech Europe \\ University Campus STeP Ri \\ Slavka Krautzeka 83/A \\ 51000 Rijeka, Croatia \\ Phone: +385 (51) 770447 \\ Fax: +385 (51) 686166 \\ www.intechopen.com
}

\author{
InTech China \\ Unit 405, Office Block, Hotel Equatorial Shanghai \\ No.65, Yan An Road (West), Shanghai, 200040, China \\ 中国上海市延安西路65号上海国际贵都大饭店办公楼 405 单元 \\ Phone: +86-21-62489820 \\ Fax: $+86-21-62489821$
}


(C) 2008 The Author(s). Licensee IntechOpen. This chapter is distributed under the terms of the Creative Commons Attribution-NonCommercialShareAlike-3.0 License, which permits use, distribution and reproduction for non-commercial purposes, provided the original is properly cited and derivative works building on this content are distributed under the same license. 